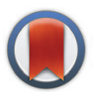

CrossMark

Cite this: Org. Chem. Front., 2015, 2, 446

DOI: $10.1039 / c 5 q 090010 g$

rsc.li/frontiers-organic

\section{Correction: Novel strategies for catalytic asymmetric synthesis of C1-chiral 1,2,3,4-tetrahydroisoquinolines and 3,4-dihydrotetrahydroisoquinolines}

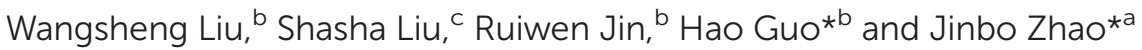 \\ Correction for 'Novel strategies for catalytic asymmetric synthesis of C1-chiral 1,2,3,4-tetrahydroisoquino- \\ lines and 3,4-dihydrotetrahydroisoquinolines' by Wangsheng Liu et al., Org. Chem. Front., 2015, DOI: \\ $10.1039 / \mathrm{c} 4 \mathrm{qo} 00294 \mathrm{f}$
}

The authors regret an error in the title and abstract with regard to one compound, i.e., "3,4-dihydrotetrahydroisoquinolines" in the title should read "dihydroisoquinolines". "3,4-dihydroisoquinoline" in the abstract should also read "dihydroisoquinoline", to avoid causing any confusion to the readers. In addition, on page 9, "THIQ 63" should read "isoquinoline derivative 63". Finally, "3,4-dihydroisoquinolines" in the Graphical Abstract should read "dihydroisoquinolines".

The Royal Society of Chemistry apologises for these errors and any consequent inconvenience to authors and readers.

\footnotetext{
${ }^{a}$ Department of Chemistry, Northeast Normal University, 5268 Renmin Road, Changchun, Jilin 130024, P. R. China. E-mail: zhaojb100@nenu.edu.cn

${ }^{b}$ Department of Chemistry, Fudan University, 220 Handan Road, Shanghai, 200433, P. R. China. E-mail: hao_guo@fudan.edu.cn

${ }^{c}$ School of Geographical Sciences, Northeast Normal University, 5268 Renmin Road, Changchun, 130024 Jilin, P. R. China
} 